\title{
Adaptación y validación española del Physical Activity Class Satisfaction Questionnaire (PACSQ)*
}

\author{
Spanish Adaptation and Validation of the Physical Activity \\ Class Satisfaction Questionnaire (PACSQ)
}

\author{
Álvaro Sicilia ** \\ ROBERTO FERRIZ \\ Universidad CEU Cardenal Herrera. Facultad de Humanidades \\ y Ciencias de la Comunicación. Elche, España
RUBÉN TRIGUEROS **** \\ RUBÉN TRIGUEROS *** \\ Universidad de Almería, España \\ DAVID GONZÁLEZ-CUTRE***** \\ Universidad Miguel Hernández de Elche, España
}

Recibido: mayo 31 de 2013 | Revisado: abril 16 de 2014 | Aceptado: abril 16 de 2014

doi:10.11144/Javeriana.UPSY13-4.ayve

Para citar este artículo: Sicilia, A., Ferriz, R. Trigueros, R., \& González-Cutre, D. (2014). Adaptación y validación española del Physical Activity Class Satisfaction Questionnaire (PACSQ). Universitas Psychologica, 13(4), 1321-1332. http://dx.doi. org/10.11144/Javeriana.UPSY13-4.ayve

* La realización de este trabajo fue posible gracias al proyecto de investigación: «Análisis de la influencia de las clases de Educación Física en la adherencia a la práctica deportiva y la adopción de hábitos de vida saludables tras finalizar la escolarización obligatoria" (Ref. DEP2010-17063), financiado por el Ministerio de Ciencia e Innovación, España.

*** Facultad de Ciencias de la Educación, Enfermería y Fisioterapia,. Universidad de Almería. España. Correo electrónico: asicilia@ual.es. Universidad CEU Cardenal Herrera. Facultad de Humanidades y Ciencias de la Comunicación. Elche (España). roberto.ferriz@uchceu.es

***** Correo electrónico: ruv_1987@hotmail.com

${ }^{* * * * *}$ Centro de Investigación del Deporte. Universidad Miguel Hernández de Elche. España. Correo electrónico:dgonzalez-cutre@umh.es

\section{RESUMEN}

El objetivo de este estudio fue validar la versión española del Physical Activity Class Satisfaction Questionnaire (PACSQ) dentro del contexto de la Educación Física (EF). La muestra estuvo compuesta por 858 estudiantes, de edades comprendidas entre 15 y 21 años. Las propiedades psicométricas del PACSQ fueron analizadas a través de varios análisis. Los resultados apoyaron el modelo de primer orden con nueve factores y el modelo de orden superior. La estructura de ambos modelos fue invariante respecto al género. La correlaciones entre las subescalas indicaron un modelo factorial relacionado, apoyando la validez de constructo de la escala. Los valores alfa de Cronbach fueron superiores a 0.7 y se obtuvieron niveles apropiados de estabilidad temporal. Los resultados de este estudio muestran evidencias de validez para la utilización del PACSQ en el contexto español.

Palabras clave

validación; propiedades psicométricas; satisfacción; Educación Física

\footnotetext{
A B S T R A C T

The purpose of this study was to validate the Spanish version of the Physical Activity Class Questionnaire (PACSQ) into the physical education (PE) context. The sample consisted of 858 students, aged between 15 to 21 years, and the psychometric properties of the PACSQ were examined through different analyses. The results supported both the first-order nine-factor model and the higher-order model. The structure of both models was invariant across gender. Correlations among the subscales indicated a related factor model supporting construct validity of the scale. Alpha values over 0.7 and suitable levels of temporal stability were obtained. The findings of this study provided validity evidences for using the PACSQ in a Spanish context. Keywords validation; psychometric properties; satisfaction; physical education
} 
La satisfacción que sienten las personas durante la realización de actividades comenzó a estudiarse en el ámbito laboral, analizándose la relación entre la motivación y la efectividad organizativa (Dunnette \& Hough, 1990). Dada precisamente su asociación con el rendimiento, la investigación de este constructo empezó a aplicarse tanto al contexto deportivo como al académico, incluyendo la Educación Física (Chelladurai \& Riemer, 1997; Duda \& Nicholls, 1992; Nicholls, Patashnick, \& Nolen, 1985; Riemer \& Chelladurai, 1998; Treasure \& Roberts, 1994, 1998).

La investigación sobre la satisfacción en el deporte y en la Educación Física (EF) ha mostrado que este constructo mantiene asociación con conductas afectivas, cognitivas y conductuales positivas para el ejercicio físico y el rendimiento escolar. De hecho, la investigación ha indicado que la satisfacción se muestra como un antecedente importante del esfuerzo para aprender, el afán de superación, la motivación por la actividad, la importancia concedida a la EF y la intención por mantenerse físicamente activo (Baena-Extremera, Granero-Gallegos, Bracho-Amador, \& Pérez-Quero, 2012; Duda \& Nicholls, 1992; Ntoumanis, 2001; Ruiz-Juan, Gómez-López, Pappous, Alacid, \& Flores, 2010). No obstante, además de ser indicada como antecedente, la satisfacción ha sido considerada como una consecuencia positiva en sí misma, en la medida en que refleja el efecto que los entornos, métodos y experiencias vividas en la clase de EF están teniendo en los estudiantes (Cervelló \& Santos-Rosa, 2000; Cunningham \& Xiang, 2008). Tal y como Chelledurai y Riemer (1997) han indicado, la satisfacción reflejaría "un estado afectivo positivo que resulta de evaluar las estructuras, procesos y resultados" (p. 135), siendo un constructo de interés para evaluar las experiencias que los estudiantes de EF experimentan en sus clases.

A pesar de la importancia que la satisfacción de los estudiantes puede tener para predecir consecuencias o evaluar la misma calidad de la enseñanza en EF, existen hasta el momento pocos instrumentos que puedan medirla. Además, mientras en el contexto deportivo han existido aproximaciones multidimensionales para definir y medir la satisfac- ción (Ortega, Jiménez, Palao, \& Sainz de Baranda, 2008; Riemer \& Chelladurai, 1998; Treasure \& Roberts, 1994, 1998), la definición de este constructo para el contexto de la EF ha sido limitada a una aproximación unidimensional. Así, el instrumento más utilizado para medir la satisfacción en las clases de EF ha sido la Intrinsic Satisfaction Classroom Scale ([ISC]; Duda \& Nicholls, 1992), que fue una adaptación del instrumento originalmente elaborado por Nicholls et al. (1985) para evaluar la satisfacción en contextos académicos. La ISC ha sido validada al contexto español con varias muestras de estudiantes adolescentes y utilizada para evaluar la satisfacción en clases de EF (Balaguer, Atienza, Castillo, Moreno, \& Duda, 1997; Castillo, Balaguer, \& Duda, 2001; Granero-Gallegos, Baena-Extremera, Pérez-Quero, Ortiz-Camacho, \& Bracho-Amador, 2012). No obstante, este instrumento se basa en una definición unidimensional de la satisfacción, en la medida en que valora el grado en que los estudiantes se divierten (p. ej., "Normalmente me divierto en las clases de EF"; "Cuando hago EF parece que el tiempo vuela") o se aburren (p. ej., "En las clases de EF normalmente me aburro"; "Deseo que la clase termine rápidamente"). De este modo, dimensiones relevantes para la satisfacción en EF (p. ej., calidad de la instrucción, relación con compañeros), las cuales han sido consideradas para otros contextos (p. ej., deportivo, laboral), podrían estar siendo omitidas.

Para atender a esta limitación, Cunningham (2007) desarrolló el Physical Activity Class Satisfaction Questionnaire (PACSQ). Este instrumento contempla una definición multidimensional de la satisfacción en clases de actividad física, a partir de la revisión de este constructo en otros contextos. El PACSQ fue desarrollado y validado originalmente por medio de tres estudios con muestras independientes de estudiantes universitarios. En el primero, Cunningham identificó diferentes dimensiones de la satisfacción, a través de la respuesta dada por 16 estudiantes a un cuestionario de preguntas abiertas, donde debían indicar las diferentes fuentes de satisfacción respecto a su participación en las clases de actividad física recibidas. Un total de ocho fuentes 
o dimensiones fueron definidas: (a) Experiencias de maestría: percepción del desarrollo o mejora de aprendizajes relacionados con habilidades físicas; (b) Desarrollo cognitivo: percepción del desarrollo o mejora de aprendizajes cognitivos, tales como conceptos básicos de la materia; (c) Éxito normativo: percepción de superioridad en comparación con los iguales, ya sea intelectual o física; (d) Interacción con los demás: ambiente o clima percibido al socializarse con otros iguales; (e) Diversión y disfrute: cuando las actividades realizadas son percibidas como entretenidas y divertidas; (f) Mejora de la salud y la condición física (CF): percepción de que las actividades de clase contribuyen al mantenimiento o mejora del estado de salud en general; (g) Experiencias recreativas: cuando la clase es percibida como estimulante y rejuvenecedora, contribuyendo tanto al bienestar físico como emocional; (f) Relajación: cuando la clase es percibida en su contribución para aliviar y liberar el estrés, desconectando de la rutina académica de otras clases. Cada una de estas ocho dimensiones fue constatada con una revisión de la literatura sobre satisfacción en diferentes contextos, incluido el deportivo y el laboral.

A partir de las ocho dimensiones extraídas del estudio cualitativo, en un segundo estudio, Cunningham (2007) redactó, con la ayuda de tres expertos, diferentes ítems para cada una de las dimensiones. Un primer análisis factorial confirmatorio (AFC) mostró que los índices de ajuste del modelo eran aceptables, aunque éstos mejoraban si dos ítems eran reubicados en otro factor. Por otro lado, los ocho factores fueron revisados y, aunque un factor de enseñanza no fue indicado por los estudiantes durante el estudio cualitativo, finalmente fue incorporado al objeto de poder evaluar la satisfacción de un factor que parece esencial en un proceso de enseñanza (la instrucción recibida por los estudiantes dentro del contexto de la clase). De este modo, un modelo final del PACSQ con nueve factores y 45 ítems fue sometido a un nuevo AFC en un tercer estudio. Los análisis mostraron unos índices de ajuste apropiados para este modelo y una consistencia interna adecuada con valores alfa de Cronbach iguales o superiores a 0.85. Finalmente, los resultados del tercer estudio mostraron evidencias de validez predictiva al correlacionar positivamente las diferentes dimensiones con cuatro consecuencias relacionadas con la satisfacción (intención de repetir clases similares, probabilidad de hablar positivamente de las clases, continuar realizando ejercicio físico en los próximos años y continuar haciendo ejercicio físico el resto de la vida). No obstante, los resultados mostraron correlaciones altas entre el factor desarrollo cognitivo y experiencias de maestría (0.91), entre mejora de la salud/CF y experiencias recreativas $(0.86)$ y entre este último y relajación (0.82). A pesar de estas correlaciones la estructura de nueve factores fue mantenida, dado que existían diferencias conceptuales y los resultados indicaron también diferencias entre los factores en cuanto a la fuerza de asociación que cada uno de ellos mantuvo con las conductas predichas en el estudio.

El instrumento desarrollado por Cunningham (2007) se presenta como una herramienta de interés, para poder evaluar en España diferentes dimensiones de la satisfacción en las clases de EF. Hasta el momento, la utilización de la ISC ha limitado la medición del constructo a una polaridad establecida entre los factores diversión-aburrimiento. Por lo tanto, el objetivo del presente estudio fue adaptar al contexto español el Physical Activity Class Satisfaction Questionnaire ([PACSQ]; Cunningham, 2007) y analizar sus propiedades psicométricas.

En primer lugar, dado que el PACSQ podría ser usado tanto para calcular la media de cada subescala como para obtener la puntuación media total de la escala, se realizaron AFC de dos modelos diferentes. En el primer modelo, se hipotetizó que los nueves factores de primer orden, los cuales representan las nueve dimensiones de la satisfacción con las clases de EF, estarían correlacionados. En el segundo modelo, se propuso una estructura con nueve factores de orden primario y un factor de orden superior denominado satisfacción. En segundo lugar, se comprobó la invarianza factorial respecto al género de los modelos propuestos. En último lugar, se analizó la consistencia interna y la estabilidad temporal del instrumento. 


\section{Método}

\section{Participantes}

En este estudio, tomaron parte 858 estudiantes de educación secundaria posobligatoria (405 chicos y 453 chicas), con edades comprendidas entre los 15 y 21 años $(M=16.72 ; D E=0.84)$, pertenecientes a once centros educativos de dos provincias españolas.

Para analizar la estabilidad temporal del PACSQ, se utilizó una segunda muestra independiente de 93 estudiantes de educación posobligatoria, con edades comprendidas entre 15 y 17 años $(M=15.94 ; D E$ $=0.38$ ), quienes completaron el instrumento en dos ocasiones, con un intervalo de tiempo de dos semanas entre la primera y segunda toma de datos.

\section{Medidas}

Cuestionario de satisfacción en las clases de Educación Física (CSCEF)

Se empleó una versión adaptada y traducida al español del Physical Activity Class Satisfaction Questionnaire ([PACSQ]; Cunningham, 2007). El cuestionario está encabezado por la sentencia: "Indica tu nivel de satisfacción con las clases de Educación Física recibidas respecto a...", y consta de 45 ítems repartidos en nueve factores (Apéndice) ${ }^{1}$. Los estudiantes debían indicar su respuesta por medio de una escala Likert del 1 (totalmente desacuerdo) al 8 (totalmente de acuerdo).

1 Nota.Se han eliminado de la versión original los siguientes ítems: un ítem del factor enseñanza: "1. La calidad de la enseñanza en general"; un ítem del factor relajación: "21. El modo en que soy capaz de relajarme durante la Educación Física"; un ítem del factor diversión y disfrute: "8. Mi disfrute en general en clase"; dos ítems del factor éxito normativo: "4. Mi rendimiento comparado con otros en la clase" y "42. Mi capacidad para superar a otros compañeros/as de clase”; dos ítems del factor experiencias de maestría: "1. La oportunidad de aprender nuevas habilidades" y "6. El grado en que mejoro mis propias habilidades"; dos ítems del factor experiencias recreativas: "11. Lo estimulante que son las clases" y "27. Cómo de motivado/a me siento durante las clases"; y tres ítems del factor interacción con otros: " 5 . La oportunidad que tengo de conocer a personas con intereses similares", "23. La oportunidad de conocer a gente nueva en las clases" y "30. Mi comunicación con el resto de compañeros/as de clase".

\section{Procedimiento}

Con el objetivo de validar el cuestionario al contexto español, se adoptó una estrategia de traducción inversa (Hambleton, 1996). Durante este proceso el cuestionario original fue vertido al español por un grupo de traductores y posteriormente otro grupo lo tradujo a su idioma original. La bondad de la traducción se juzgó en función del grado de coincidencia con la versión original. La versión obtenida fue analizada por tres expertos (Lynn, 1986) en EF, de tal modo que se garantizara que los ítems estuvieran bien diseñados para medir el constructo que quería medirse y que retuviera el significado original.

Una vez traducido el instrumento se contactó con los responsables de los once centros educativos participantes, que a su vez derivaron la colaboración en el profesorado de EF, a los que también se les informó del objetivo de la investigación y solicitó su colaboración. Al alumnado menor de edad se le requirió una autorización de sus padres para su participación. La administración del cuestionario se realizó bajo la supervisión de al menos un miembro del grupo de investigación, que explicó la forma de cumplimentarlo y solventó las dudas que se plantearon. El tiempo estimado para completar el cuestionario estuvo en torno a 20 minutos.

\section{Análisis de datos}

Para determinar la validez y fiabilidad del PACSQ en el contexto español, se realizó un análisis de sus propiedades psicométricas. En primer lugar, se realizaron dos AFC para testar tanto la estructura de nueve factores como la estructura de un modelo que englobara la satisfacción como factor de orden superior. En segundo lugar, se llevaron a cabo análisis multigrupo para analizar la invarianza de los modelos respecto al género. Finalmente, se proporcionaron los estadísticos descriptivos y se evaluó la fiabilidad del instrumento a través de análisis de consistencia interna (alfa de Cronbach) y un análisis de estabilidad temporal (índice de correlación intraclase [CCI]). Para los análisis de datos, se utilizaron los paquetes estadísticos SPSS 19.0 y AMOS 19.0. 
Puesto que el coeficiente de Mardia fue alto (755.327), para los diferentes AFC se utilizó el método de estimación de máxima verosimilitud junto con el procedimiento de bootstrapping. Los estimadores no se vieron afectados por la falta de normalidad, por lo que fueron considerados robustos (Byrne, 2001). Con el objetivo de aceptar o rechazar los modelos testados, se utilizó un conjunto de varios índices de ajuste: $\chi^{2} / g l$, CFI (Comparative Fit Index), IFI (Incremental Fit Index), RMSEA (Root
Mean Square Error of Approximation) más su intervalo de confianza (IC) al $90 \%$ y SRMR (Standardized Root Mean Square Residual). Dado que el $\chi^{2}$ es muy sensible al tamaño muestral (Jöreskog \& Sörbom, 1993), se empleó el $\chi^{2} / g l$, considerándose aceptables valores inferiores a 5 (Bentler, 1989). Los índices incrementales (CFI e IFI) muestran un buen ajuste con valores de 0.9 o superiores (Schumacker \& Lomax, 1996), mientras que los índices de error (RMSEA y SRMR) se consideran aceptables con

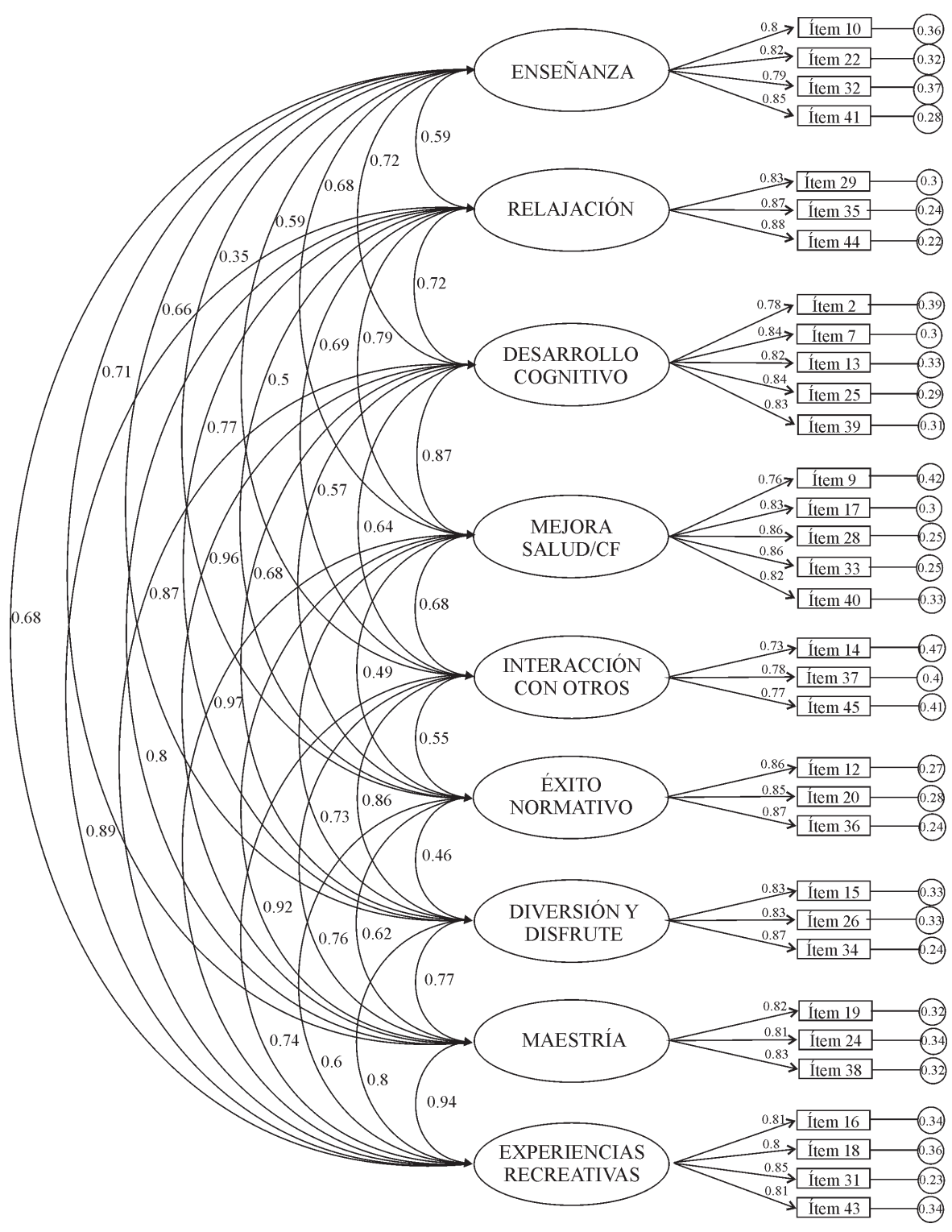

Figura 1. Análisis factorial confirmatorio del PACSQ adaptado a la EF. Las elipses representan los factores y los rectángulos los diferentes ítems. Las varianzas residuales se muestran en los círculos pequeños.

Fuente: elaboración propia 
valores iguales o menores de 0.08 (Browne \& $\mathrm{Cu}$ deck, 1993; Hu \& Bentler, 1999).

\section{Resultados}

\section{Análisis factorial confirmatorio}

Inicialmente, se evaluó la estructura de un modelo de 45 ítems y nueve factores, presentando el modelo los siguientes índices de ajuste: $\chi^{2}(909, N=858)$ $=4235.93, p=0.001 ; \chi^{2} / g l=4.66 ; \mathrm{CFI}=0.9 ; \mathrm{IFI}$ $=0.9 ; \operatorname{RMSEA}=0.065(\mathrm{IC} 90 \%=0.063-0.067)$; SRMR $=0.0519$. No obstante, los índices de modificación sugerían posibles mejoras y, tras analizar la matriz residual estandarizada de covarianzas, se observó que los valores residuales de algunos ítems correlacionaban con los valores residuales de otros ítems y estaban asociados a residuales estandarizados > |2.00| (Jöreskog \& Sörbom, 1984). Del modelo fueron excluidos un total de doce ítems. En concreto, se eliminó un ítem de tres de los factores (enseñanza, diversión y disfrute, y relajación); dos ítems de otros tres factores (experiencias recreati- vas, experiencias de maestría y éxito normativo), y tres ítems del factor interacción con los otros (Apéndice). Excluyendo estos doce ítems el ajuste del modelo a los datos mejoró ostensiblemente: $\chi^{2}$ $(459, N=858)=1616.76, p=0.001 ; \chi^{2} / g l=3.52 ;$ $\mathrm{CFI}=0.95 ; \mathrm{IFI}=0.95 ; \mathrm{RMSEA}=0.054(\mathrm{IC} 90 \%$ $=0.051-0.057) ; \mathrm{SRMR}=0.03$.

En este modelo final (Figura 1), se observó que los valores residuales estandarizados de todos los ítems estuvieron situados por debajo de dos en valores absolutos y los pesos de regresión estandarizados fueron estadísticamente significativos $(p<$ 0.001 ), oscilando entre 0.73 y 0.88 . No obstante, se obtuvieron correlaciones elevadas entre varios factores, siendo las más elevadas las correlaciones entre los factores experiencias recreativas y mejora de la salud (0.97), experiencias de maestría y desarrollo cognitivo (0.96), experiencias de maestría y experiencias recreativas (0.94) y experiencias de maestría y mejora de la salud/CF (0.92).

Debido a las altas correlaciones entre algunos factores, se evaluó la sostenibilidad de diversos modelos alternativos con ocho factores (Tabla 1).

TABLA 1

Índices de ajuste para modelos alternativos de ocho factores del PASCQ

\begin{tabular}{lccccccc}
\hline & $\chi 2$ & $\mathrm{gl}$ & $\chi 2 / \mathrm{gl}$ & CFI & IFI & SRMR & RMSEA (IC 90\%) \\
\hline F7 + F1(ER y MS/CF) & 1757.67 & 467 & 3.76 & 0.95 & 0.95 & 0.033 & $0.057(0.054-0.06)$ \\
F7 + F1(EM y DG) & 1742.13 & 467 & 3.73 & 0.92 & 0.95 & 0.033 & $0.056(0.054-0.059)$ \\
F7 + F1(EM y ER) & 1800.58 & 467 & 3.86 & 0.91 & 0.94 & 0.032 & $0.058(0.055-0.061)$ \\
F7 + F1(EM y MS/CF) & 1857.01 & 467 & 3.96 & 0.94 & 0.94 & 0.034 & $0.059(0.056-0.062)$ \\
F7 + F1(ER y R) & 1996.5 & 467 & 4.28 & 0.94 & 0.94 & 0.035 & $0.062(0.059-0.065)$ \\
F7 + F1(ER y DG) & 2151.45 & 467 & 4.61 & 0.93 & 0.93 & 0.037 & $0.065(0.062-0.068)$ \\
F7 + F1(DGy MS/CF) & 2144.67 & 467 & 4.59 & 0.93 & 0.93 & 0.035 & $0.065(0.062-0.068)$ \\
F7 + F1(DD y R) & 1749.41 & 467 & 3.75 & 0.95 & 0.95 & 0.032 & $0.057(0.054-0.059)$ \\
F7 + F1(ER y DD) & 2252.89 & 467 & 4.82 & 0.92 & 0.93 & 0.039 & $0.067(0.064-0.07)$ \\
F7 + F1(EM y R) & 2193.49 & 467 & 4.7 & 0.93 & 0.93 & 0.036 & $0.066(0.063-0.068)$ \\
\hline
\end{tabular}

Nota. $\mathrm{E}=$ Enseñanza; $\mathrm{R}=$ Relajación; $\mathrm{DG}=$ Desarrollo Cognitivo; MS/CF = Mejora Salud y Condición Física; IO = Interacción con Otros; EN = Éxito Normativo; DD = Diversión y Disfrute; EM = Experiencias de Maestría; ER = Experiencias Recreativas.

Fuente: elaboración propia 
Para estos modelos alternativos, dos de las subescalas que mostraron correlaciones altas fueron combinadas para formar un solo factor que se uniera al modelo junto con las restantes subescalas. Un total de diez modelos alternativos fueron testados y comparados con el modelo de nueve factores. Los diez modelos alternativos mostraron índices de ajuste peores que el modelo de nueve factores, lo que apoyaría la validez discriminante de las subescalas.

Una vez determinado el modelo que mejor se ajustaba a los datos, se procedió a testar un modelo de orden superior (los nueve factores de primer orden convergiendo en un factor de segundo orden denominado Satisfacción). Los índices de ajuste de este modelo fueron ligeramente peores, aunque en general aceptables: $\chi^{2}(486, N=858)=$ 2213.1, $p=0.001, \chi^{2} / g l=4.55 ; \mathrm{CFI}=0.93 ; \mathrm{IFI}=$ 0.93; RMSEA $=0.064($ IC 90\% $=0.062-0.067)$; SRMR $=0.044$. Todos los pesos de regresión estandarizados fueron significativos $(p<0.001)$, siendo de 0.72 para enseñanza, 0.86 relajación, 0.91 para desarrollo cognitivo, 0.95 para mejora de la salud, 0.78 para interacción con otros, 0.59 éxito normativo, 0.81 para diversión y disfrute, 0.97 para experiencias de maestría y 0.99 experiencias recreativas.

TABLA 2

Análisis multigrupo de invarianza respecto al género

\section{Análisis de invarianza respecto al género}

Para comprobar si la estructura factorial del modelo de nueve factores y del modelo de un factor de orden superior eran invariantes respecto al género, se procedió a realizar análisis multigrupo. En la Tabla 2 , se muestran los diversos índices de ajuste para los cuatro y seis modelos comparados, respectivamente. Para el modelo de nueve factores, no se encontraron diferencias significativas entre el modelo sin restricciones (modelo 1) y el modelo de invarianza en los pesos de medida (modelo 2). Por su parte, los resultados sí revelaron diferencias ente el modelo 1 y el modelo de covarianzas estructurales invariantes (modelo 3) y el modelo de residuos de medida invariantes (modelo 4). La ausencia de diferencias significativas entre el modelo 1 y el modelo 2 supone un criterio mínimo para aceptar que la estructura del modelo es invariante respecto al género (Byrne, Shavelson, \& Muthén, 1989; Marsh, 1993). Respecto al modelo de orden superior, no se encontraron diferencias significativas entre el modelo sin restricciones (modelo 1) y el modelo de invarianza en los pesos de medida (modelo 2), el modelo de pesos estructurales invariantes (modelo 3 ) y el modelo de covarianzas estructurales invariantes (modelo 4). Los resultados mostraron diferencias significativas

\begin{tabular}{lccccccccc}
\hline \multicolumn{8}{c}{ Modelo de nueve factores de orden primario } \\
\hline Modelos & $\chi 2$ & $\mathrm{gl}$ & $\chi 2 / \mathrm{gl}$ & $\Delta \chi^{2}$ & $\Delta \mathrm{gl}$ & $\mathrm{CFI}$ & $\mathrm{IFI}$ & $\mathrm{RMSEA}(\mathrm{IC} 90 \%)$ & SRMR \\
\hline Modelo 1 & 2318.93 & 918 & 2.53 & - & - & 0.94 & 0.94 & $0.042(0.4-0.44)$ & 0.04 \\
Modelo 2 & 2340.63 & 942 & 2.49 & 21.706 & 24 & 0.94 & 0.94 & $0.042(0.4-0.44)$ & 0.039 \\
Modelo 3 & 2430.47 & 987 & 2.46 & $111.539^{* *}$ & 69 & 0.94 & 0.94 & $0.041(0.39-0.43)$ & 0.046 \\
Modelo 4 & 2493.76 & 1020 & 2.45 & $174.831^{* *}$ & 102 & 0.94 & 0.94 & $0.041(0.39-0.43)$ & 0.047 \\
\hline \multicolumn{7}{c}{} & \multicolumn{7}{c}{ Modelo de un factor de orden superior } \\
\hline Modelos & $\chi 2$ & $\mathrm{gl}$ & $\chi 2 / \mathrm{gl}$ & $\Delta \chi^{2}$ & $\Delta \mathrm{gl}$ & $\mathrm{CFI}$ & $\mathrm{IFI}$ & $\mathrm{RMSEA}(\mathrm{IC} 90 \%)$ & SRMR \\
\hline Modelo 1 & 2954.28 & 972 & 3.04 & - & - & 0.92 & 0.92 & $0.049(0.47-0.51)$ & 0.053 \\
Modelo 2 & 2975.86 & 996 & 2.98 & 21.579 & 24 & 0.92 & 0.92 & $0.048(0.46-0.5)$ & 0.053 \\
Modelo 3 & 2991.04 & 1004 & 2.98 & 36.762 & 32 & 0.92 & 0.92 & $0.048(0.46-0.5)$ & 0.054 \\
Modelo 4 & 2992.77 & 1005 & 2.98 & 38.491 & 33 & 0.92 & 0.92 & $0.048(0.46-0.5)$ & 0.056 \\
Modelo 5 & 3023.7 & 1014 & 2.98 & $69.422^{* *}$ & 42 & 0.92 & 0.92 & $0.048(0.46-0.5)$ & 0.057 \\
Modelo 6 & 3082.2 & 1047 & 2.94 & $127.92 * *$ & 75 & 0.92 & 0.92 & $0.048(0.46-0.5)$ & 0.058 \\
\hline
\end{tabular}

$* * p<0.01$.

Fuente: elaboración propia 
entre el modelo 1 y el modelo de residuos estructurales invariantes (modelo 5) y el modelo de residuos de medida invariantes (modelo 6). Estos resultados apoyan también la invarianza respecto al género para el modelo de orden superior.

\section{Estadísticos descriptivos, análisis de consistencia interna y estabilidad temporal}

Como puede observarse en la Tabla 3, las medias de los factores de satisfacción estuvieron por encima de la puntuación media de la escala, obteniendo la puntuación más alta el factor diversión y disfrute $(M=5.7)$ y la más baja el factor éxito normativo $(\mathrm{M}=4.34)$.

Para analizar la fiabilidad de la escala, se llevó a cabo un análisis de consistencia interna y un análisis de estabilidad temporal. El primer análisis reveló un valor alfa de Cronbach de 0.89 para la subescala de enseñanza; 0.9 para relajación; 0.91 para desarrollo cognitivo; 0.92 para mejora de la salud/CF; 0.8 para interacción con otros; 0.89 para éxito normativo; 0.88 para diversión y disfrute; 0.86 para experiencias de maestría y 0.89 para experiencias recreativas. Por otro lado, el análisis de estabilidad temporal se realizó con una muestra independiente, calculándose las medias y desviaciones típicas en cada una de las dos tomas realizadas, así como los coeficientes de correlación intraclase (CCI) y su intervalo de confianza
(IC). Los resultados para cada una de las subescalas del PACSQ se muestran en la Tabla 3.

\section{Discusión}

El objetivo de este estudio fue proporcionar evidencias de la validez factorial, consistencia interna y estabilidad temporal del PACSQ (Cunningham, 2007) en el contexto español. Los resultados muestran que el PACSQ es un instrumento válido y fiable para medir la satisfacción en las clases de EF de un modo multidimensional. Además, los resultados indican que la estructura factorial del instrumento se mantiene estable para chicos y chicas. Hasta el momento, la investigación realizada en España había informado de diferencias en satisfacción entre chicos y chicas (Baena-Extremera et al., 2012; Castillo et al., 2001), sin embargo, estos trabajos no habían presentado evidencias de que los instrumentos utilizados permitieran hacer tales comparaciones. Por lo tanto, los resultados de invarianza del PACSQ son interesantes en la medida en que permiten en el futuro utilizar el PACSQ para realizar comparaciones de la satisfacción en EF respecto al género. No obstante, los resultados del estudio sugieren algunas cuestiones que deberían ser atendidas en futuros trabajos.

En primer lugar, los resultados proporcionan apoyo para la validez factorial de un modelo de 33 ítems y

TABLA 3

Descriptivos y Estabilidad Temporal del PASCQ

\begin{tabular}{|c|c|c|c|c|c|c|c|}
\hline \multirow{2}{*}{ Factor } & \multicolumn{2}{|c|}{ MG } & \multicolumn{2}{|c|}{ MI (Toma 1) } & \multicolumn{2}{|c|}{ MI (Toma 2) } & \multirow[t]{2}{*}{$\mathrm{CCI}$} \\
\hline & $\mathrm{M}$ & DT & $\mathrm{M}$ & DT & $\mathrm{M}$ & DT & \\
\hline Enseñanza & 5.14 & 1.77 & 4.1 & 1.77 & 3.95 & 1.53 & $0.84(\mathrm{IC}=0.76-0.9)$ \\
\hline Relajación & 5.33 & 1.83 & 4.81 & 1.89 & 4.89 & 1.76 & $0.76(\mathrm{IC}=0.64-0.84)$ \\
\hline D. Cognitivo & 4.91 & 1.49 & 4.07 & 1.48 & 4.14 & 1.41 & $0.82(\mathrm{IC}=0.73-0.88)$ \\
\hline Mejora Salud/CF & 5.10 & 1.6 & 4.13 & 1.53 & 4.15 & 1.51 & $0.83(\mathrm{IC}=0.74-0.89)$ \\
\hline Interacción Otros & 5.51 & 1.5 & 4.9 & 1.5 & 4.91 & 1.51 & $0.73(\mathrm{IC}=0.59-0.82)$ \\
\hline Éxito Normativo & 4.34 & 1.8 & 4.07 & 1.91 & 4.29 & 1.86 & $0.79(\mathrm{IC}=0.69-0.86)$ \\
\hline Diversión/Disfrute & 5.70 & 1.6 & 5.25 & 1.59 & 5.17 & 1.59 & $0.81(\mathrm{IC}=0.71-0.88)$ \\
\hline Exp. Maestría & 5.16 & 1.54 & 4.45 & 1.54 & 4.29 & 1.44 & $0.78(\mathrm{IC}=0.67-0.86)$ \\
\hline Exp. Recreativas & 4.95 & 1.63 & 3.83 & 1.63 & 4.11 & 1.57 & $0.77(\mathrm{IC}=0.65-0.85)$ \\
\hline Satisfacción Global & 5.13 & 1.34 & 4.4 & 1.28 & 4.43 & 1.33 & $0.86(\mathrm{IC}=0.79-0.91)$ \\
\hline
\end{tabular}

Nota. $\mathrm{MG}=$ Muestra Global; MI = Muestra Independiente.

Fuente: elaboración propia 
nueve factores del PACSQ. El modelo presentó unos índices de ajuste aceptables, y el instrumento mostró una adecuada consistencia interna y estabilidad temporal, con valores superiores a 0.7 (Cicchetti, 1994; Nunnally, 1978). En línea con los resultados mostrados por Cunningham (2007), en este estudio se encontraron también correlaciones elevadas entre algunas subescalas. Sin embargo, a pesar de esto, diferentes AFC revelaron mejores índices de ajuste en el modelo con nueve factores que en modelos alternativos de ocho factores, lo que aporta evidencias de la validez discriminante de las subescalas. Tal y como sugiere Cunningham, todos los factores del PACSQ explican diferentes fuentes de satisfacción para el alumnado y, en este sentido, presentan conceptualmente claras diferencias. Por ejemplo, los factores experiencias de maestría y desarrollo cognitivo comparten una fuente común al hacer referencia ambos a la mejora del aprendizaje percibido por el estudiante, sin embargo, mientras que el primero se refiere a los aprendizajes de habilidades físicas, el segundo engloba aprendizajes relacionadas con estrategias mentales.

Por lo tanto, es probable que en el contexto de la EF las diferentes fuentes de satisfacción compartan antecedentes similares y esto explique la fuerte asociación que existe entre varias de las subescalas. De hecho, es probable que en EF un aprendizaje de estrategias cognitivas lleve asociado una mejora en los aprendizajes procedimentales, fruto de tener una mejor comprensión mental de los mecanismos que desarrollan la acción motriz. Igualmente, es lógico pensar que una percepción de mejora de los aprendizajes, especialmente cuando implica aprendizajes procedimentales que perfeccionan las habilidades físicas, esté asociada a una percepción de la mejora de la salud y condición física y, en general, a una sensación de bienestar emocional. No obstante, aunque las diferentes subescalas parecen mantener diferencias conceptuales claras sería interesante que futuros trabajos aportaran más evidencias para determinar si los estudiantes son capaces de discernir entre las diferentes fuentes de satisfacción definidas por el PACSQ.

En segundo lugar, los resultados del AFC apoyaron también la estructura de un modelo de orden superior que representa la satisfacción en EF. Desde el punto de vista metodológico este modelo apoya el uso de la medida global de la satisfacción a partir de la media de sus nueve dimensiones. Esta medida global puede ser interesante en modelos complejos donde se necesite relacionar la satisfacción en EF con otras variables. No obstante, en este modelo la dimensión éxito normativo mostró un peso de regresión inferior al resto de las dimensiones. Además, al igual que en el estudio de Cunningham (2007), la media de esta dimensión alcanzó valores inferiores al resto de dimensiones que conforman el PACSQ. Cunningham incorporó el factor éxito normativo considerando el trabajo realizado por Treasure y Roberts $(1994,1998)$ en el contexto deportivo. Sin embargo, los resultados de este estudio indican que en el contexto de la $\mathrm{EF}$, donde los estudiantes suelen percibir un clima más orientado a la tarea (autorreferente) que al ego (normativo) (Cervelló \& Santos-Rosa, 2000; González-Cutre, Sicilia, Moreno, \& Fernández-Balboa, 2009; Sicilia, Moreno, \& Rojas, 2008), el hecho de apreciar que uno tiene más habilidades que los compañeros o que realiza las actividades mejor que los demás, pudiera ser una fuente de satisfacción menos importante.

Aunque los resultados de este estudio proporcionan apoyo psicométrico para el PACSQ, se deben indicar algunas limitaciones. En primer lugar, la muestra del estudio ha sido no probabilística y, consiguientemente, los resultados no pueden ser generalizados a otros grupos de estudiantes. Además, la validación de un instrumento debería ser un proceso continuado y, en este sentido, futuros trabajos deberían explorar la estructura factorial de la escala en otras muestras de estudiantes con diferentes características (p. ej., edad, nivel educativo, nivel de satisfacción en clase). En segundo lugar, de cara a mostrar evidencias de la validez predictiva del instrumento los trabajos en el futuro podrían considerar variables que actúen de antecedentes o consecuentes de la satisfacción y analizar la asociación del constructo con estas variables. La relación de las diferentes subescalas del PACSQ con otras variables podría reforzar además la validez discriminante entre las diferentes dimensiones. Finalmente, el análisis multigrupo ha mostrado evidencias de 
que el instrumento puede ser utilizado para comparar la satisfacción entre chicos y chicas, sin embargo, futuros trabajos deberían determinar si también puede ser utilizado para establecer diferencias en la satisfacción del alumnado en función de otras variables (p. ej., tipo de centro, nivel educativo, optatividad curricular).

En resumen, los resultados de este estudio apoyan el PACSQ como un instrumento válido y fiable para medir de forma multidimensional la satisfacción en las clases de EF. Cunningham (2007) creó el PACSQ para determinar el grado en que los estudiantes universitarios estaban satisfechos con los diferentes programas de actividad física contemplados en su currículo. A través de este estudio se han mostrado evidencias de que el instrumento puede también ser utilizado para valorar la satisfacción de los estudiantes en el contexto de la EF escolar. De este modo, el profesorado y directores de centros educativos en España pueden utilizar la escala para evaluar la efectividad de los programas de enseñanza.

\section{Refrencias}

Baena-Extremera, A., Granero-Gallegos, A., BrachoAmador, C., \& Pérez-Quero, F. J. (2012). Spanish version of the sport satisfaction instrument (SSI) adapted to physical education. Revista de Psicodidáctica, 17 (2), 377-395.

Balaguer, I., Atienza, F. L., Castillo, I., Moreno, Y., \& Duda, J. L. (1997, septiembre). Factorial structure of measures of satisfaction/interest in sport and classroom in the case of Spanish adolescents. Trabajo presentado en la 4th European Conference of Psychological Assessment, Lisboa, Portugal.

Bentler, P. M. (1989). EQS structural equations program manual. Los Angeles: BMDP Statistical Software.

Browne, M. W., \& Cudeck, R. (1993). Alternative ways of assessing model fit. En K. Bollen \& J. Long (Eds.), Testing structural equation models (pp. 136162). Newbury Park, CA: Sage.

Byrne, B. M. (2001). Structural equation modeling with Amos: Basic concepts, applications, and programming. Mahwah, NJ: Erlbaum.
Byrne, B. M., Shavelson, R. J., \& Muthén, B. (1989). Testing for the equivalence of factor covariance and means structures: The issue of partial measurement invariance. Psychological Bulletin, 105, 456-466.

Castillo, I., Balaguer, I., \& Duda, J. L. (2001). Perspectivas de meta de los adolescentes en el contexto académico. Psicothema, 13(1), 79-86.

Cervelló, E., \& Santos-Rosa, F. (2000). Motivación en las clases de Educación Física: Un estudio de la perspectiva de las metas de logro en el contexto educativo. Revista de Psicología del Deporte, 9(1-2), 51-70.

Chelladurai, P., \& Riemer, H. A. (1997). A classification of the facets of athlete satisfaction. Journal of Sport Management, 11, 133-159.

Cicchetti, D. V. (1994). Guidelines, criteria, and rules of thumb for evaluating normed and standardized assessment instruments in psychology. Psychological Assessment, 6(4), 284-290.

Cunningham, G. B. (2007). Development of the physical activity class satisfaction questionnaire (PACSQ). Measurement in Physical Education and Exercise Science, 11(3), 161-176.

Cunningham, G. B., \& Xiang, P. (2008). Testing the mediating role of perceived motivational climate in the relationship between achievement goals and satisfaction: Are these relationships invariant across sex? Journal of Teaching in Physical Education, 27(2), 192-204.

Duda, J. L., \& Nicholls, J. G. (1992). Dimensions of achievement motivation in schoolwork and sport. Journal of Educational Psychology, 84(3), 290-299.

Dunnette, M. D., \& Hough, L. M. (1990). Handbook of industrial and organizational psychology. Palo Alto, CA: Consulting Psychologists Press.

González-Cutre, D., Sicilia, A., Moreno, J. A., \& Fernández-Balboa, J. M. (2009). Dispositional flow in physical education: Relationships with motivational climate, social goals, and perceived competence. Journal of Teaching in Physical Education, 28(4), 422-440.

Granero-Gallegos, A., Baena-Extremera, A., Pérez-Quero, F. J., Ortiz-Camacho, M. M., \& Bracho-Amador, C. (2012). Analysis of motivational profiles of satisfaction and importance of physical education 
in high school adolescents. Journal of Sports Science and Medicine, 11(4), 614-623.

Hambleton, R. K. (1996). Adaptación de tests para su uso en diferentes idiomas y culturas: fuentes de error, posibles soluciones y directrices prácticas. En J. Muñiz (Ed.), Psicometría (pp. 207-238). Madrid: Universitas.

Hu, L., \& Bentler, P. M. (1999). Cutoff criteria for fit indexes in covariance structure analysis: Conventional criteria versus new alternatives. Structural Equation Modeling, 6(1), 1-55.

Jöreskog, K. G., \& Sörbom, D. (1984). LISREL-IV user's guide (3.a ed.). Mooresville, IN: Scientific Software International Inc.

Jöreskog, K. G., \& Sörbom, D. (1993). LISREL 8: Structural equation modeling with the SIMPLIS command language. Chicago, IL: Scientific Software International Inc.

Lynn, M. R. (1986). Determination and quantification of content validity. Nursing Research, 35(6), 382-385.

Marsh, H. W. (1993). The multidimensional structure of physical fitness: Invariance over gender and age. Research Quarterly for Exercise and Sport, 64(3), 256-273.

Nicholls, J. G., Patashnick, M., \& Nolen, S. B. (1985). Adolescents' theories of education. Journal of Educational Psychology, 77(6), 683-692.

Ntoumanis, N. (2001). A self-determination approach to the understanding of motivation in physical education. British Journal of Educational Psychology, 71(2), 225-242.
Nunnally, J. C. (1978). Psychometric theory. New York: McGraw-Hill.

Ortega, E., Jiménez, J. M., Palao, J. M., \& Sainz de Baranda, P. (2008). Diseño y validación de un cuestionario para valorar las preferencias y satisfacciones en jóvenes jugadores de baloncesto. Cuadernos de Psicología del Deporte, 8(2), 39-58.

Riemer, H. A., \& Chelladurai, P. (1998). Development of the athelete satisfaction questionnaire (ASQ). Journal of Sport and Exercise Psychology, 20(2), 127-156.

Ruiz-Juan, F., Gómez-López, M., Pappous, A., Alacid, F., \& FloreS, G. (2010). Dispositional goal orientation, beliefs about the causes of success and intrinsic satisfaction in young elite paddlers. Sport, Physical Education and Recreation, 26, 123-136.

Schumacker, R. E., \& Lomax, R. G. (1996). A beginner's guide to structural equation modeling. Mahwah, $\mathrm{NJ}$ : Erlbaum.

Sicilia, A., Moreno, J. A., \& Rojas, A. J. (2008). Motivation profiles and flow in physical education lessons. Perceptual and Motor Skills, 106(2), 473-494.

Treasure, D. C., \& Roberts, G. C. (1994). Cognitive and affective concomitants of task and ego orientations during middle school years. Journal of Sport and Exercise Psychology, 16(1), 15-28.

Treasure, D. C., \& Roberts, G. C. (1998). Relationship between female adolescents' achievement goal orientations, perceptions of motivational climate, belief about success and sources of satisfaction in basketball. International Journal of Sport Psychology, 29(3), 211-230. 


\section{Apéndice}

\section{Enseñanza}

10. La claridad de las explicaciones del profesor.

22. El entusiasmo del profesor durante las clases.

32. La empatía que el profesor muestra hacia los estudiantes en las clases.

41. La habilidad del profesor para comunicar con eficiencia el contenido de la materia.

\section{Relajación}

29. El modo en que mi mente puede desconectar.

35. El modo en que la Educación Física me ayuda a liberarme del estrés.

44. El modo en que las clases de Educación Física me ayudan a relajarme.

\section{Desarrollo cognitivo}

2. Lo que aprendo respecto a los aspectos técnicos de la Educación Física.

7. Cuánto aprendo sobre las diversas estrategias utilizadas en Educación Física.

13. Lo que aprendo sobre el contenido básico de la Educación Física.

25. Los conocimientos que adquiero sobre los fundamentos de la Educación Física.

39. El grado en que aprendo los conceptos básicos de la Educación Física.

\section{Mejora de la salud/CF}

9. La mejoría de mi salud debido a las clases.

17. El entrenamiento físico que recibo en las clases.

28. El desarrollo de una mejor condición física debido a las clases.

33. La contribución de las clases a mi estado general de salud.

40. El progreso para lograr un cuerpo más sano durante las clases.

\section{Interacción con otros}

14. Las relaciones que tengo con otros en las clases.

37. La oportunidad que tengo de socializarme con otros.

45. El ambiente o clima social en general de las clases.

\section{Éxito normativo}

12. La superioridad de mis habilidades en comparación con el resto de clase.

20. Mi capacidad de hacerlo mejor que otros compañeros/as de clase.

36. Mis habilidades comparadas con las de otros compañeros/as de clase.

\section{Diversión y disfrute}

15. La diversión que tengo en clase.

26. Las experiencias agradables que tengo en clase.

34. Lo bien que me lo paso en clase.

\section{Experiencias de maestría}

19. Cuánto aprendo acerca de cómo realizar mejor actividad física o deporte.

24. Mi mejoría en la ejecución de habilidades.

38. Mi oportunidad de practicar nuevas habilidades.

\section{Experiencias recreativas}

16. La manera en que la Educación Física contribuye a mi bienestar emocional.

18. Cómo de rejuvenecido me siento como resultado de las clases.

31. El modo en que las clases me hacen sentir revitalizado/a.

43. El esfuerzo físico estimulante durante las clases. 